\title{
WHY HUMANITY IN MEDICAL EDUCATION
}

\author{
Chief Editor, Birat Journal of Health Sciences
}

\author{
Associate Prof. Dr. Hem Sagar Rimal, MBBS, MD (Paediatrics), MPH, Fellowship in Development Paediatrics \\ Department of Paediatrics \\ Birat Medical College \& Teaching Hospital \\ Tankisinuwari, Morang, Nepal
}

\section{Citation}

Rimal HS. Why Humanity in Medical Education. BJHS 2017, 2(3)4: 236-237

DOI: http://dx.doi.org/10.3126/bjhs.v2i3.18932

Medicine is a field which does not discriminate. It is a subject that is governed by the sole essence of being human. The anatomy that applies to the rich, applies to the poor. The biochemistry that controls one religion is the same that controls another. In the end, the most important thing that matters in medicine is humanity - The essence of being human. The art of understanding others is humanity. The ability to love, care for and show empathy towards others is humanity. No matter how skillful a doctor may be, to be able to successfully show that skill, he must have the means necessary to deliver his skills. No matter how hard working a doctor may be, she must be able to project that hard work towards her patients in a way that the patient feels safe and comforted. You see, In spite of what other brilliant qualities that a health professional may have, if they are not able to project those qualities with humanity and love, they will never reach the heights of patient care that their potential warranted.

The current state of medical education reflects the lack of proper and holistic patient care worldwide and more so in our region. Our curriculum is based solely on the scientific teachings of medicine. It doesn't fully encompass the psychological aspect of medicine and more importantly the human aspect of medicine. Medical ethics, patient management, communication, diagnosis delivery etc. are all huge facets of what it is to be a doctor, but are all overlooked while teaching. Humanities are a crucial part of the medical profession. The foundation of medicine lies in the science of healing. Healing is a broad term that encompasses not just physical recovery, but the wide array of minor recoveries that are made by the patient to reintegrate into society. That opens up an ocean of steps that should be taken up by a health professional to facilitate the patient's process of rehabilitation. It is integral to understand the patient's perspective when all decisions are made. Everyone who has practiced medicine has faced these situations before. Say there is a patient who can't afford some expensive yet completely necessary treatment, or you are left to counsel the family of a patient who has just died of a seemingly incurable disease. While no-one wants to be in that situation we have to face it - that sort of situation happens quite often in the course of practicing medicine and it is one of if not the most draining element of the practice altogether. Very few are born with an intrinsic knack for helping others and for the rest of us - Don't worry, like any skill humanities can be learnt.

Humanities are social disciplines that study social interaction and culture. Their integration in medical education would instill essential characteristics- (i) Empathy and (ii) Humanism in up and coming doctors. Some may argue that these are soft studies, mere distractions from the core subjects of Anatomy, Physiology etc. but that is a comparison of a measurable entity with an immeasurable one. This view helps to maintain this stubborn misconception. And yes, this endeavor doesn't have any large economic prospects nor should you expect any notable effects in patient survival or recovery. But it sure will ameliorate the impact that a medical institution has on society and that is very important in the grand scheme of things.

Humanities can and should be learnt by everyone in the medical profession. Its incorporation into the curriculum would mean that the quality of medical service would only go up and up. The Medical practice is different from other consumer services in that it is extremely personal, with patients often venting out all of their emotions that have accumulated due to the stresses of being sick or having a loved one in that situation. Humanities would in such scenarios help practitioners to dish out valid advice balancing reassurance with realism. Medicine exceeds just knowledge. Like any discipline it is equal parts 'skills' and 'attitude' too.

Since we are realizing a need of a "humane" doctor with understanding, assisted by interpretive ability and insight, and governed by ethical sensitivity to apply this scientific evidence and skills to the individual patient, our medical education system probably needs a paradigm shift incorporating the component of humanities in medical education. ${ }^{2}$ This should be a longitudinal process that begins 
from the day he /she enters medical school. Inclusion of topics like history of medicine, doctor patient's relationship, medical ethics, informed consents, breaking bad news etc. from very early on is essential. ${ }^{3}$ This helps to develop a concept that "The best physicians are also philosophers" something which was expressed by the Famed Physician Galen. It's amazing to realize that our present shortcomings in this regard were already being thought of in the $2^{\text {nd }}$ century AD. So it's often wise to take a evaluative look in the history books in order to make legitimate and informed decisions for the future. ${ }^{4}$ Teaching humane aspects and attributes of a good doctor is not easy job to do through didactic lecture only. We can introduce cineeducation by selecting movies and students can write their reflection after watching them. Similarly writing narratives about their encounter with different kind of patients in different contexts would enhance their understanding of humanity. Interactive group discussion, seminar on doctor patient relationship and case study on the role of empathy are other modalities. With appropriate role modeling and inspiration we can, without any specific scheduled classes instill the value of humanity in the student and that most probably is the best scenario we can hope at the moment.

\section{REFERENCES}

1. Reidar Pederson. Empathy Development in Medical Education-A Critical review; Medical Teacher 2010;32;593-600.

2. Britta M Thompson, Jerry B Vannatta, Laura E Scobey, Mark Fergeson, Humanities research group and Sheila M Crow. Providing context for Medical School basic science curriculum: The importance of the humanities; Medical Teacher 2016; 38; 82-87.

3. Edward C. Halperin. Preserving the Humanities in Medical Education; Medical Teacher 2010; 32; 76-79.

4. Lester Liao, Department of Pediatrics, University of Alberta. Opening our eyes to a critical approach to medicine: The humanities in medical education ; Medical Teacher $2017 ; 39 ; 220-221$. 